\title{
Management of Acute Symptomatic Hydronephrosis of Pregnancy by Postural Drainage
}

\author{
Sudhanshu Chitale ${ }^{*}, 1,2$ and Vinayak R. Chitale ${ }^{1,2}$ \\ ${ }^{I}$ Dr. V.M. Medical College General Hospital, Solapur, India \\ ${ }^{2}$ Chitale Clinic, 165, D, Rly. Lines, Solapur, 413001, India
}

\begin{abstract}
Introduction \& Objectives: Asymptomatic hydronephrosis is found in $>90 \%$ of pregnant women, only a small proportion become symptomatic. Non-surgical management is the treatment of choice. In refractory cases surgical intervention is indicated.

We present our experience with conservative management of acute symptomatic hydronephrosis of pregnancy by facilitating postural drainage of the obstructed symptomatic kidney.

Material \& Methods: 1750 women with full term pregnancy were managed in the unit over 5 yrs. 130 (7.4\%) presented with unilateral loin pain and were in the third trimester of pregnancy at presentation (20@28 weeks, 58@30 weeks, and 52@ 32 weeks). 68 / 130 (52.3\%) were multi-parous. Age range was 26-38 yrs.
\end{abstract}

$115(88.5 \%)$ presented with right-sided loin pain and $15(11.5 \%)$ had pain in the left loin. There was no evidence of incipient urinary tract infection (UTI). Ultrasound scan (USS) confirmed presence of ipsilateral mild to moderate hydronephrosis with no evidence of renal / ureteric calculus.

All were refractory to routine enteral or parenteral analgesia over $72 \mathrm{hrs}$.

They were managed in a semi-prone position while in bed, with the affected side up and non-dependant. Head end of their bed was kept raised by $10^{\circ}$ throughout this period of conservative management; initiated in the hospital and continued at home until term.

Results: 121 of the 130 women (93.1\%) had symptomatic improvement. None required regular analgesia and went to term without further intervention i.e. insertion of nephrostomy / stenting. Follow-up USS at three months post-partum revealed complete resolution of hydronephrosis.

Conclusions: Postural drainage of the kidney is highly effective in the management of acute symptomatic hydronephrosis of pregnancy. In the majority of cases (93\%), if used diligently, it will help prevent the need for invasive uro-radiological intervention.

Keywords: Hydronephrosis, pregnancy, symptomatic, postural drainage, conservative.

\section{INTRODUCTION}

Dilatation of the upper urinary tract involving the pelvicalyceal system and the upper two thirds of ureter is a physiological development during pregnancy. It is being detected earlier than expected and at earlier times than previously reported however; over $90 \%$ of women remain asymptomatic with it throughout their pregnancy. Conservative measures are advocated in managing women with symptomatic hydronephrosis of pregnancy and intervention indicated only in those failing to respond to conservative management. We present our experience of managing such patients conservatively with particular emphasis on postural drainage of the affected kidney based on the well established and accepted fact that kidneys drain better while in prone position.

*Address correspondence to this author at the Norfolk \& Norwich University Hospital, Colney Lane, Norwich, NR4 7UY, UK; Fax: 0044 1603 287884; E-mail: chitalenorwich@aol.com

\section{MATERIALS AND METHODS}

Over a period of 5 yrs., 1750 women presenting with full term pregnancy were managed in our local obstetrics units. $130(7.4 \%)$ women presented with unilateral loin pain. All 130 were in the third trimester of pregnancy (20@28 weeks, 58@30weeks, and 52@32 weeks).68 of them were multiparous. Age range was 26-38 yrs (Mean age: 30.2 yrs). Clinical data for these patients was collected prospectively with stringent follow up for upto three months to confirm successful outcome of their conservative management. 115 women presented with right sided pain and 15 had pain in the left loin. Their blood picture and midstream urine (MSU) examination revealed no evidence of incipient urinary tract infection (UTI). None had significant impairment of renal function. Ultrasound scan (USS) confirmed presence of mild to moderate hydronephrosis on the affected side with appearances not dissimilar to any asymptomatic hydronephrosis of pregnancy and no evidence of urinary calculus disease. Randomisation to analgesia or no analgesia + postural drainage was not performed for ethical reasons. 
Regular enteral and parenteral analgesia was offered to all women for up to $72 \mathrm{hrs}$ but, failed to improve their presenting symptom of loin pain. 9 out of 130 women $(6.9 \%)$ had severe refractory pain or became systemically unwell to warrant intervention such as insertion of a ureteric stent or percutaneous nephrostomy (PCN) and were excluded from the final analysis.

All the remaining 121 symptomatic women were managed with bed rest on a tilted bed with its head end raised by $10^{\circ}$ using wooden blocks (Fig. 1) and the woman lying on the bed in a semi prone position with the affected side up (Fig. 2) throughout the period of conservative management up to full term. It was initially instituted in the hospital setting with a mean inpatient stay of three days and subsequently continued with similar arrangements at home.
During this period however, all women remained fully ambulatory and adopted the semi-prone position on the head end-elevated sloping bed only while resting both during the day and night.

\section{RESULTS}

All 121 women in our series had substantial symptomatic improvement with this regime aimed at encouraging postural drainage of the obstructed kidney. No one required further regular analgesia as long as they followed this regime and went on to term without intervention in the form of $\mathrm{JJ}$ stenting or nephrostomy. Follow-up USS at three months postpartum revealed complete resolution of hydronephrosis and no evidence of urinary calculi. During this observational period to term, these women served as their own controls

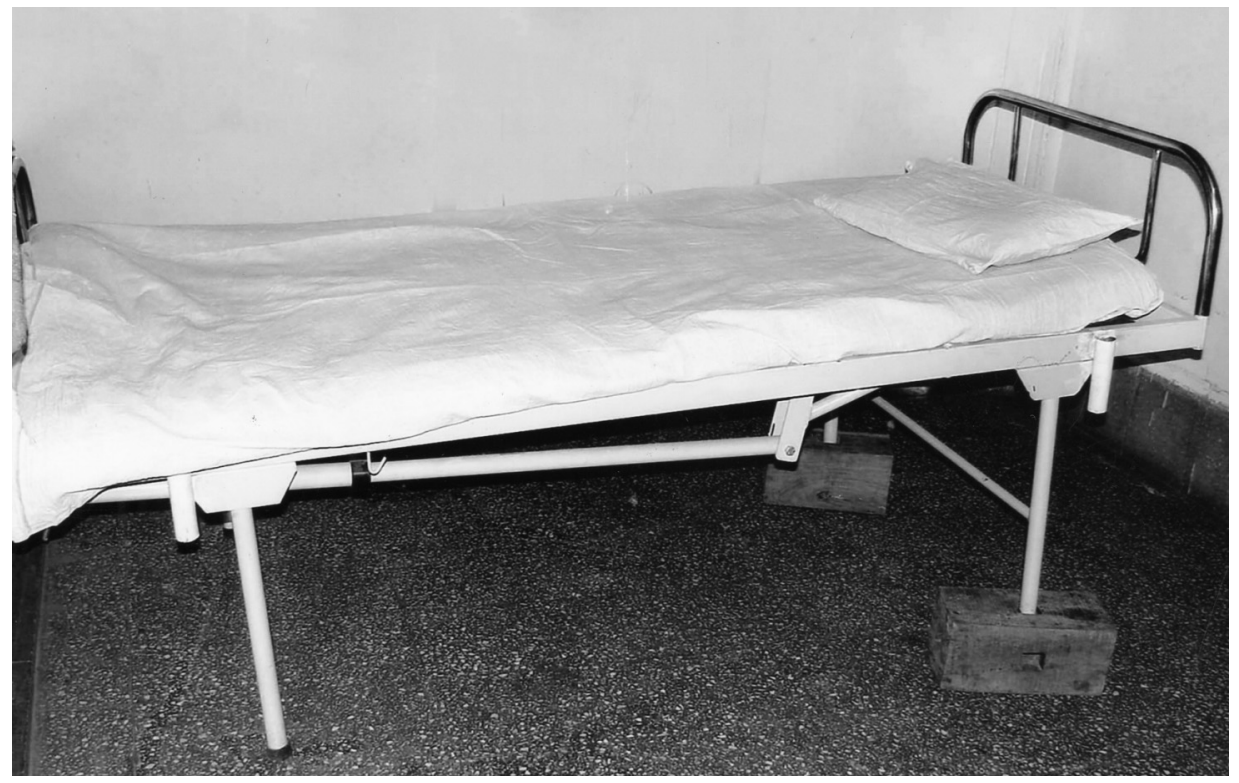

Fig. (1). Head end of the bed elevated using wooden blocks.

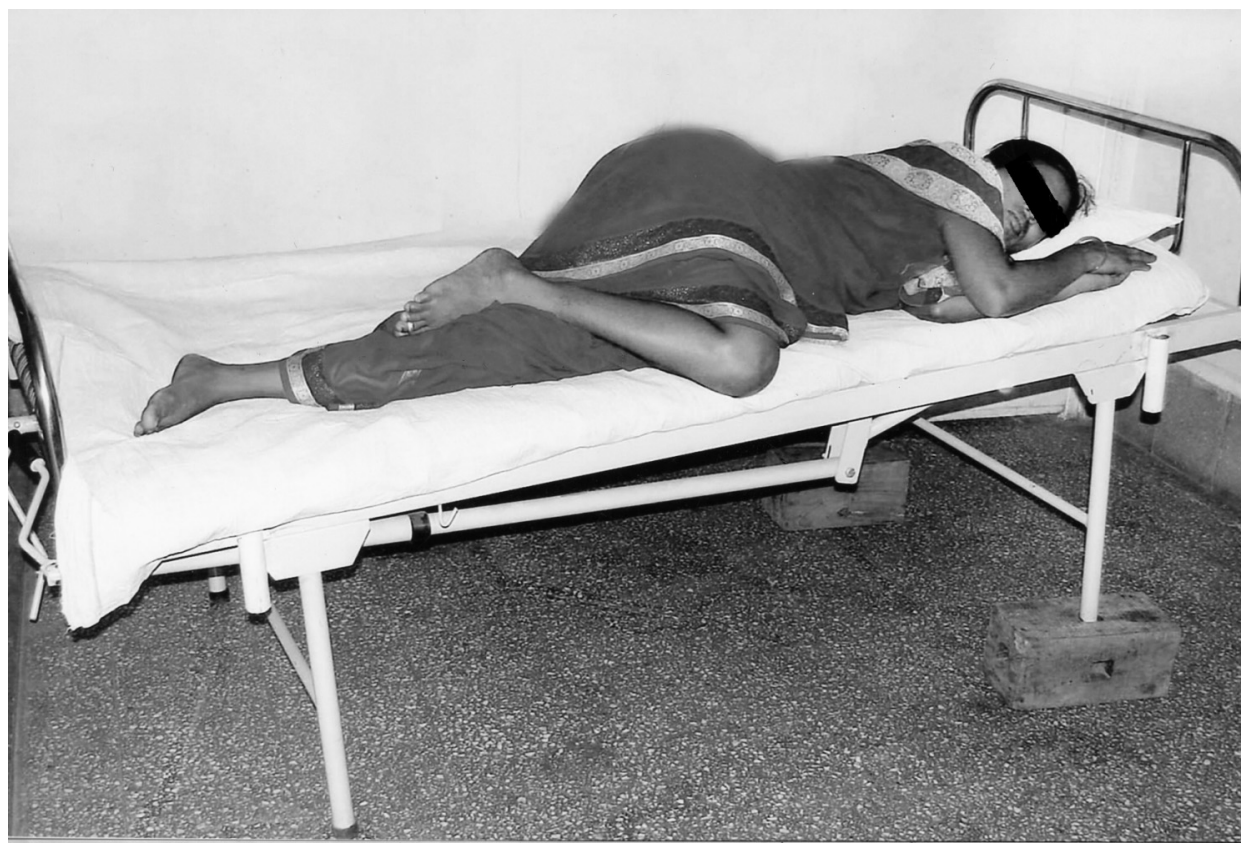

Fig. (2). Patient with symptomatic hydronephrosis of pregnancy on the right hand side lying semi-prone on the head-end elevated bed with her affected (right) side up. 
because they experienced loin pain whenever they did not adopt the recommended semi-prone position during resting but had pain relief following change of position and at times required an occasional dose of oral analgesia. All women maintained adequate urine output throughout this regime independent of the position while at rest and this could be attributed to the non-obstructed normally functioning contralateral renal unit.

\section{CONCLUSION}

This prospective observational study has shown that postural drainage of the obstructed kidney is highly effective in the management of acute symptomatic hydronephrosis of pregnancy. In the majority of cases (93\%), if used diligently, it will help prevent the need for invasive uro-radiological intervention.

\section{DISCUSSION}

Hydronephrosis is a common finding during pregnancy affecting over $90 \%$ of pregnant women [1,2] with some degree of dilatation of the pelvi-calyceal system and upper two thirds of the ureter. It develops from as early as week 6 to week 10 of gestation and such onset of dilatation is seen earlier than previously reported on routine prenatal ultrasound scans (USS) however, the degree of obstruction can be quite variable for the asymptomatic patient [3]. These changes are a consequence of: increased renal blood flow (up to $75 \%$ by term) and the smooth muscle relaxant effects of progesterone as well as mechanical obstruction from the enlarging foetus and uterus, hence predominantly seen on the right side but not in pelvic kidneys and those transplanted into ileal conduits. Hydronephrosis of pregnancy is not seen in quadrupeds such as dogs and cats where the gravid uterus in a dependant position falls away from the ureters. We believe the same mechanism works in human beings when the woman adapts a semi-prone position with the affected side up and head end elevated, thereby allowing the gravid uterus to fall forward away from the retroperitoneal ureters and taking the mechanical pressure off them, particularly the affected ureter and allowing it to drain more freely. Ureteric drainage is further helped by the head-up position of the bed.

In contrast to asymptomatic hydronephrosis, symptomatic hydronephrosis would prompt a search for other causes of obstruction namely renal/ureteric stones and an USS may help diagnosis with variable reports about its sensitivity and specificity in different series $[4,5]$. Magnetic resonance urography (MRU) has been shown to be of value in evaluating painful hydronephrosis of pregnancy as it shows characteristic and differing urographic appearances in physiological and calculous obstruction [6]. Magnetic resonance excretory urography (MREU) is another novel technique that gives equivalent functional and additional anatomical information compared to isotope renography and is more accurate than Doppler ultrasound in evaluating ureteric obstruction in pregnancy [7].

Most studies reported in the literature recommend conservative approach $[1,8,9]$ for the management of hydronephrosis of pregnancy including for renal colic in pregnancy [4] and aggressive methods such as insertion of JJ ureteral stent reserved for those not responding to conservative measures $[1,4,8-10]$. Insertion of percutaneous nephrostostomy (PCN) has been advocated in patients with symptomatic hydronephrosis associated with spontaneous extravasation of urine [2], urosepsis and azotemia [11] while ureteroscopic removal of stone only in selected cases [1].

In our series, the incidence of symptomatic hydronephrosis of pregnancy was slightly higher $(7.4 \%)$ compared to other series $(0.2 \%)$ [8]. which may simply be the result of variation in the patient population with regards to patient age, gestational age at presentation, parity, ethnicity, pain threshold and we need a larger prospective study to compare the effects of these factors. Obstruction due to calculus disease was ruled out with reasonable confidence based on USS findings and clinical, haematological picture as well as urine microbiology. Those cases in which calculus obstruction was suspected were further investigated with a single film IVU but are not included in this study.

All women responded very well to the postural drainage regime of spending most of their time in bed lying semiprone with the affected (mostly right) side up. A clear understanding of the problem and full cooperation on part of the patient was vital for a successful outcome avoiding any active intervention up to term in this scenario.

It is important that the patient is carefully monitored throughout this period and at the slightest indication of recurrent/persistent symptoms failing to respond to postural drainage or indeed clinical evidence of UTI involving upper tracts, appropriate measures such as antibiotic therapy and / or ultrasound guided drainage of obstructed $+/$ - infected kidney or internal JJ stenting are instituted as spontaneous rupture of hydronephrotic kidney during pregnancy has been reported as a rare but possible complication [12-14].

We believe that this simple conservative approach in patients showing satisfactory response will avoid unnecessary interventions in the majority of cases and also avoid consequential prolongation of inpatient hospital stay and inherent increase in distress. It will substantially reduce the overall cost of management which has significant implications in the developing world, both in the private and state funded hospitals.

\section{REFERENCES}

[1] Navalon VP, Sanchez BF, Pallas CY, et al. Symptomatic hydronephrosis during pregnancy. Arch Esp Urol 2005; 58: 977-82.

[2] Ferguson T, Bechtel W. Hydronephrosis of pregnancy. Am Fam Physician 1991; 43: 2135-7.

[3] Fried AM. Hydronephrosis of pregnancy: ultrasonographic study and classification of asymptomatic women. Am J Obstet Gynecol 1979; 135: 1066-70.

[4] Parulkar BG, Hopkins TB, Wollin MR, Howard PJ Jr, Lal A. Renal colic during pregnancy: a case for conservative treatment. J Urol 1998; 159: 365-8.

[5] Stothers L, Lee LM. Renal colic in pregnancy. J Urol 1992; 148 : 1383-7.

[6] Spencer JA, Chahal R, Kelly A, Taylor K, Eardley I, Lloyd SN. Evaluation of painful hydronephrosis in pregnancy: magnetic resonance urographic patterns in physiological dilatation versus calculous obstruction. J Urol 2004; 171: 256-60.

[7] Spencer JA, Tomlinson AJ, Weston MJ, Lloyd SN. Early report: comparison of breath-hold MR excretory urography, Doppler ultrasound and isotope renography in evaluation of symptomatic hydronephrosis in pregnancy. Clin Radiol 2000; 55: 446-53.

[8] Fainaru O, Almong B, Gamzu R, Lessing JB, Kupferminc M. The management of symptomatic hydronephrosis in pregnancy. BJOG 2002; 109: 1385-7. 
[9] Zwergel T, Lindenmeir T, Wullich B. Management of acute hydronephrosis in pregnancy by ureteral stenting. Eur Urol 1996; 29: 292-7.

[10] Sahin H, Bircan MK, Yayla M. JJS application in acute symptomatic hydronephrosis in pregnancy. Int J Gynaecol Obstet 1997; 59: 141-2.

[11] van Sonnenberg E, Casola G, Talner LB, Wittich GR, Varney RR, D'Agostino HB. Symptomatic renal obstruction or urosepsis during pregnancy: treatment by sonographically guided percutaneous nephrostomy. Am J Roentgenol 1992; 158: 91-4.
[12] Dhabuwala CB, Riehle RA Jr. Spontaneous rupture of a hydronephrotic kidney during pregnancy. Urology 1984; 24: 591-4.

[13] Hwang SS, Park YH, Lee CB, Jung YJ. Spontaneous rupture of a hydronephrotic kidney during pregnancy: value of serial sonography. J Clin Ultrasound 2000; 28: 258-60.

[14] Meyers SJ, Lee RV, Munschauer RW. Dilatation and nontraumatic rupture of the urinary tract during pregnancy: a review. Obstet Gynecol 1985; 66: 809-15.

(C) Chitale and Chitale; Licensee Bentham Open.

This is an open access article licensed under the terms of the Creative Commons Attribution Non-Commercial License (http://creativecommons.org/licenses/by$\mathrm{nc} / 3.0 /$ ) which permits unrestricted, non-commercial use, distribution and reproduction in any medium, provided the work is properly cited. 